\title{
Voltage-induced Shapiro steps in a superconducting multi-terminal structure
}

\author{
J.C. Cuevas ${ }^{1,2,3}$ and H. Pothier ${ }^{4}$ \\ ${ }^{1}$ Departamento de Física Teórica de la Materia Condensada, \\ Universidad Autónoma de Madrid, E-28049 Madrid, Spain \\ ${ }^{2}$ Institut für Theoretische Festkörperphysik, Universität Karlsruhe, D-76128 Karlsruhe, Germany \\ ${ }^{3}$ Forschungszentrum Karlsruhe, Institut für Nanotechnologie, D-76021 Karlsruhe, Germany and \\ ${ }^{4}$ Quantronics Group, Service de Physique de l'État Condensé, \\ DRECAM, CEA-Saclay, 91191 Gif-sur-Yvette, France
}

(Dated: April 10, 2018)

\begin{abstract}
When a superconducting tunnel junction at a finite voltage is irradiated with microwaves, the interplay between the alternating Josephson current and the ac field gives rise to steps in the dc current known as Shapiro steps. In this work we predict that in a mesoscopic structure connected to several superconducting terminals one can induce Shapiro-like steps in the absence of any external radiation simply by tuning the voltages of the leads. To illustrate this effect we make quantitative predictions for a three-terminal structure which comprises a diffusive superconductor-normal metalsuperconductor (SNS) junction and a tunneling probe, a set-up which can be realized experimentally.
\end{abstract}

PACS numbers: $74.45 .+\mathrm{c}, 74.50 .+\mathrm{r}, 73.23 .-\mathrm{b}$

\section{INTRODUCTION}

In 1962 Josephson predicted that a tunnel junction between two superconductors at a finite bias $V$ would sustain an alternating current with frequency $2 \mathrm{eV} / \hbar \frac{1}{1}$ Josephson also argued that the alternating current would be frequency-modulated by an applied rf field and this would lead to steps in the dc current-voltage characteristics at bias voltages given by $V=m h \nu / 2 e$, where $\nu$ is the frequency of the field and $m$ is an integer. In 1963 Shapiro shone microwaves onto a junction and observed the predicted steps, $\stackrel{2}{=}$ which since then are referred to as Shapiro steps. This experiment constituted the first proof of ac Josephson effect and also paved the way for applications of this effect. $\frac{3}{}$

The Shapiro steps are a consequence of the interplay between an ac Josephson current and a microwave signal. The observation of Shapiro steps does not necessarily require an external microwave generator: when two Josephson junctions are electromagnetically coupled like in the classical experiment of Giaever, $\stackrel{4}{=}$ a voltage-biased junction can be used as a microwave source and induce Shapiro steps in the current-voltage characteristics (I-V) of the other one. More subtle coupling schemes have been achieved, in which two superconducting weak links placed a few micrometers apart share a common electrode. Shapiro-like steps were then observed in the I-Vs, which were attributed to the phase-locking of the Josephson frequencies when the dc voltages on two bridges are approximately matched. $\underline{\underline{5}}$ Although there is no rigorous microscopic theory of this non-equilibrium effect, it is usually attributed to the diffusion of the quasiparticle charge imbalance generated in and around the weak links $\underline{\underline{6}}$ Very recently, it has been predicted that one could also generate Shapiro steps by coupling a tunnel Josephson junction to a mechanical oscillator $\stackrel{?}{?}$

In this work we predict that a different type of Shapirolike steps can appear in a single multi-terminal supercon-

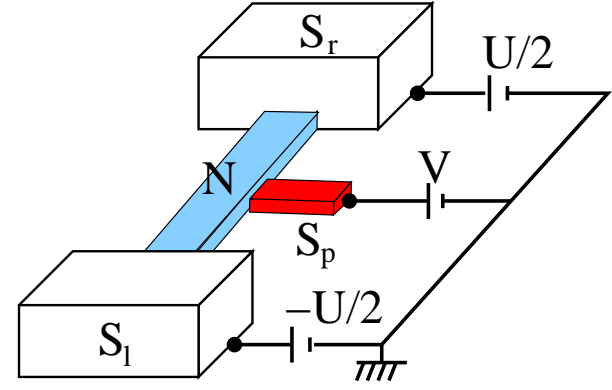

FIG. 1: (Color online) Schematic representation of the setup: a metallic wire $(\mathrm{N})$ is connected at its ends to superconducting reservoirs $\mathrm{S}_{l}$ and $\mathrm{S}_{r}$, biased at potentials $-U / 2$ and $U / 2$, respectively. An additional superconducting probe $\left(\mathrm{S}_{p}\right)$ at a potential $\mathrm{V}$ is attached to the wire through a tunnel junction.

ducting structure without any external radiation. The idea goes as follows. Inspired by the experiment of Ref. [8], let us consider the three-terminal structure shown in Fig. 1 It is formed by a diffusive SNS junction, and a superconducting tunneling probe $\mathrm{S}_{p}$ attached to the normal wire. If one applies a potential difference $U$ across the wire, ac currents with a frequency $2 e U / h$ and its harmonics will flow along the $\mathrm{N}$ wire. If a potential $V$ is now applied to the probe electrode, it will generate ac currents with Josephson frequencies $2 e(V \pm U / 2) / h$. The interference between these ac currents which both involve the quasiparticles in the diffusive wire gives rise to contributions to the dc current through the interface $\mathrm{N}-\mathrm{S}_{p}$, which in this setup appear as Shapiro steps at discrete voltages $V=m U / 2$, where $m$ is an odd integer. Notice that in this case the steps appear due to the nonequilibrium state created in the diffusive wire. In this region the energy distribution function of quasiparticles is driven to oscillate at different frequencies by the dc voltages of the leads and the Shapiro steps are a result of the 
beating of these internal Josephson frequencies. As illustrated below, this effect is described quantitatively using the quasiclassical theory of superconductivity for diffusive systems, in particular time-dependent Usadel equations. Finally, it is important to emphasize that these voltage-induced Shapiro steps may appear in a great variety of mesoscopic structures and, as explained below, it constitutes a valuable way to directly test the ac Josephson effect.

The rest of the paper is organized as follows. In section II we remind the basic physics of diffusive SNS junctions at finite bias. Section III is devoted to the description of the theoretical formalism and contains the main technical results. In section IV we discuss the numerical results for both the current-voltage characteristics of the tunneling probe and the dependence of the height of the voltage-induced Shapiro steps on the different system parameters. Finally, in section V we briefly summarize the main conclusions of this work.

\section{BASIC PHYSICS OF DIFFUSIVE SNS JUNCTIONS}

The effect described above is a consequence of the nonequilibrium properties of diffusive SNS junctions. The physics of these junctions is the result of the interplay between the proximity effect in the normal wire and the occurrence of multiple Andreev reflections (MARs). The proximity effect is the modification of the properties of a normal metal in contact with a superconductor and it has been extensively studied in diffusive hybrid nanostructures. $\frac{9}{}$ MARs are the tunneling processes that dominate the subgap transport in SNS junctions. Here, successive Andreev reflections at both S electrodes lead to a progressive rise of the quasiparticle energy until its energy exceeds the gap energy, $\Delta \stackrel{10}{\underline{10}}$ The interplay between proximity effect and MARs in diffusive SNS junctions gives rise to a rich variety of phenomena. Thus for instance, the conductance exhibits a very peculiar subgap structure $\underline{11}$ Dubos et al $\stackrel{12}{\underline{1}}$ studied the ac Josephson effect by shining microwaves onto a junction, and they observed the appearance of fractional Shapiro steps, which is a signature of a non-sinusoidal supercurrent-phase relation. On the other hand, using the setup of Fig. 1 Pierre et $a l \stackrel{8}{\text { }}$ measured the non-equilibrium distribution function in a long silver wire, where the proximity effect was negligible. They showed that this function exhibits several steps, which is a manifestation of MARs.

The consequences of the interplay between proximity effect and coherent MARs in the dissipative current has been addressed theoretically only very recently by using the time-dependent Usadel equations. $\underline{13}$ This theory describes the crossover from the short junction regime $(L \ll \xi=\sqrt{\hbar D / \Delta}), \stackrel{14}{\underline{t}}$ where $L$ is the wire length, $\xi$ is the superconducting coherence length and $D$ the diffusion constant, to the incoherent limit $(L \gg \xi) \stackrel{15}{\underline{15}}$ The intermediate regime is the relevant one for the observa- tion of the voltage-induced Shapiro steps.

\section{CALCULATION OF THE VOLTAGE-INDUCED SHAPIRO STEPS}

We consider the structure depicted in Fig. 1, where the position of the tunneling probe along the wire is denoted by $x\left(x_{l}=0\right.$ and $\left.x_{r}=L\right)$. We assume that the three superconductors $\left(\mathrm{S}_{l}, \mathrm{~S}_{r}\right.$ and $\left.\mathrm{S}_{p}\right)$ have the same energy gap $\Delta$. Our goal is the calculation of the current $I(V)$ through the probe junction $\mathrm{S}_{p}$ when a voltage $U$ is applied across the wire. We assume the $\mathrm{N}-\mathrm{S}_{l, r}$ interfaces to be fully transparent and neglect phase-breaking phenomena.

To solve our problem we use the quasiclassical theory of superconductivity for diffusive systems. $\frac{16}{2}$ This theory is formulated in terms of momentum averaged Green functions $\check{\mathbf{G}}\left(\mathbf{R}, t, t^{\prime}\right)$ which depend on position $\mathbf{R}$ and two time arguments. These propagators are $2 \times 2$ matrices in Keldysh space $\left(^{(}\right)$, where each entry is a $2 \times 2$ matrix in electron-hole space $\left(^{\wedge}\right)$ :

$$
\check{\mathbf{G}}=\left(\begin{array}{cc}
\hat{G}^{R} & \hat{G}^{K} \\
0 & \hat{G}^{A}
\end{array}\right) ; \quad \hat{G}^{R}=\left(\begin{array}{cc}
\mathcal{G}^{R} & \mathcal{F}^{R} \\
\tilde{\mathcal{F}}^{R} & \tilde{\mathcal{G}}^{R}
\end{array}\right) .
$$

The Green functions for the left (l) and right (r) leads and for the probe $(\mathrm{p})$ electrode can be written as $\check{\mathbf{G}}_{j}\left(t, t^{\prime}\right)=$ $e^{-i \phi_{j}(t) \hat{\tau}_{3} / 2 \hbar} \check{\mathbf{G}}_{0}\left(t-t^{\prime}\right) e^{i \phi_{j}\left(t^{\prime}\right) \hat{\tau}_{3} / 2 \hbar}$, where $\phi_{j}(t)$ is the phase of the order parameter of the electrode $j=l, r, p$ given by $\partial \phi_{j}(t) / \partial t=2 \mu_{j} / \hbar$, where the chemical potentials are $\mu_{l}=-e U / 2, \mu_{r}=e U / 2$ and $\mu_{p}=e V$. Here, $\check{\mathbf{G}}_{0}(t)$ is the equilibrium bulk Green function of a BCS superconductor. We now transform to energy representation, in which the propagator $\check{\mathbf{G}}\left(\mathbf{R}, \epsilon, \epsilon^{\prime}\right)$ depends on two energy arguments. It satisfies the non-stationary Usadel equation, which in the $\mathrm{N}$ region reads

$$
\frac{\hbar D}{\pi} \nabla(\check{\mathbf{G}} \circ \nabla \check{\mathbf{G}})+\epsilon \hat{\tau}_{3} \check{\mathbf{G}}-\check{\mathbf{G}} \hat{\tau}_{3} \epsilon^{\prime}=0
$$

where $\hat{\tau}_{3}$ is the Pauli matrix in electron-hole space. The convolution product $\circ$ is defined as $(\check{\mathbf{A}} \circ \check{\mathbf{B}})\left(\epsilon, \epsilon^{\prime}\right)=$ $\int d \epsilon_{1} \check{\mathbf{A}}\left(\epsilon, \epsilon_{1}\right) \check{\mathbf{B}}\left(\epsilon_{1}, \epsilon^{\prime}\right)$. Equation (22) is supplemented by the normalization condition $\check{\mathbf{G}} \circ \check{\mathbf{G}}=-\pi^{2} \delta\left(\epsilon-\epsilon^{\prime}\right) \check{\mathbf{1}}$. Due to the finite bias $U$, to solve Eq. (2) is a formidable task, which is explained in detail in Ref. [13]. What matters for our discussion is that the Green functions in the wire adopt the form:

$$
\check{\mathbf{G}}\left(\mathbf{R}, \epsilon, \epsilon^{\prime}\right)=\sum_{m} \check{\mathbf{G}}_{0, m}(\epsilon) \delta\left(\epsilon_{m}-\epsilon^{\prime}\right),
$$

where $\epsilon_{m}=\epsilon+m e U$. Here, $m$ is an even integer for the diagonal components of the Green functions in Nambu space and an odd integer for the off-diagonal ones. Eq. (3) means, in particular, that the energy distribution function and the density of states in the normal wire oscillates with the Josepshon frequency $2 e U / h$ and 
its harmonics. These oscillations result in a parametric pumping of the $\mathrm{N}-\mathrm{S}_{p}$ junction and hence to Shapiro steps.

Assuming that the electrode $S_{p}$ is weakly coupled to the $\mathrm{N}$ wire, we can express the time-dependent current $I(V, t)$ through the tunnel probe up to first order in the tunneling conductance $G_{T}$ as follows 17

$$
I(V, t)=\left(\frac{G_{T}}{8 \pi e}\right) \int d t_{1} \operatorname{Tr}\left\{\hat{\tau}_{3}\left[\check{\mathbf{G}}_{w}\left(t, t_{1}\right), \check{\mathbf{G}}_{p}\left(t_{1}, t\right)\right]^{K}\right\},
$$

where $\check{\mathbf{G}}_{w}$ is the Green function of the wire at the position of the probe junction and $\check{\mathbf{G}}_{p}$ is the Green function of the probe electrode, i.e. a bulk BCS Green funtion. Using Eqs. (3/4) it is easy to show that

$$
I(V, t)=\sum_{m=-\infty}^{\infty} \sum_{n=-1}^{1} I_{n}^{m}(V) e^{i(n \phi+2 n e V t / \hbar+m e U t / \hbar)}
$$

where $\phi$ the dc part of the phase difference. From Eq. (5), one can distinguish two contributions to the dc current, which we shall simply denote as $I$. First, there is a background current $I_{B}=I_{0}^{0}$ that contributes at every voltage $V$. More important, there is a series of contributions at discrete voltages $V_{m}=m U / 2$ with $m$ odd, ${ }^{18}$ which are given by $I_{\text {Shapiro }}(\phi)=\sum_{m>0}\left(I_{1}^{-m} e^{i \phi}+I_{-1}^{m} e^{-i \phi}\right) \delta(V-$ $\left.V_{m}\right)$, where $I_{1}^{-m}=\left(I_{-1}^{m}\right)^{*}$. Thus, Shapiro-like resonances are induced every time the probe voltage $V$ is an odd multiple of $U / 2$. From Eq. (4), one can show that the ac current components in Eq. (5) can be expressed as

$$
\begin{array}{r}
I_{1}^{m}=\left(\frac{G_{T}}{8 \pi^{2} e}\right) \int_{-\infty}^{\infty} d \epsilon\left\{\left[\mathcal{F}_{w}^{R}\right]_{0, m}(\epsilon) \tilde{\mathcal{F}}_{p}^{K}\left(\epsilon_{m}+e V\right)\right. \\
\left.+\left[\mathcal{F}_{w}^{K}\right]_{0, m}(\epsilon) \tilde{\mathcal{F}}_{p}^{A}\left(\epsilon_{m}+e V\right)\right\} .
\end{array}
$$

Here, $\left[\mathcal{F}_{w}^{R, K}\right]_{0, m}(\epsilon)$ are Fourier components of the anomalous Green functions induced in the normal wire by proximity effect. These components contain the information of ac Josephson effect in the SNS junction and depend on $L, U$ and $x$. Obviously, when $L \gg \xi$ these components vanish and in turn the Shapiro steps. 19 The functions $\tilde{\mathcal{F}}_{p}^{A, K}(\epsilon)$ are the bulk Green functions of the electrode $\mathrm{S}_{p}$ given by $\tilde{\mathcal{F}}_{p}^{A}(\epsilon)=-\pi \Delta / \sqrt{\Delta^{2}-\left(\epsilon-i 0^{+}\right)^{2}}$ and $\tilde{\mathcal{F}}_{p}^{K}(\epsilon)=-2 i \operatorname{Im}\left\{\tilde{\mathcal{F}}_{p}^{A}(\epsilon)\right\} \tanh (\beta \epsilon / 2)$, where $\beta=1 / k_{B} T$ is the inverse of the temperature.

For completeness let us also mention that the background current can be approximated by

$I_{B}=\left(\frac{G_{T}}{e}\right) \int_{-\infty}^{\infty} d \epsilon \rho_{p}(\epsilon-e V) \rho_{w}(\epsilon)\left[f(\epsilon-e V)-f_{w}(\epsilon)\right]$,

where $f(\epsilon)$ is the Fermi function, $f_{w}(\epsilon)$ is the dc part of the distribution function of the $\mathrm{N}$ wire at the position of $S_{p}, \rho_{p}(\epsilon)$ is the bulk BCS density of states and $\rho_{w}(\epsilon, U)=$ $\operatorname{Im}\left\{\left[\mathcal{G}_{w}^{A}\right]_{0,0}\right\} / \pi$ is the non-equilibrium spectral density in the normal wire.

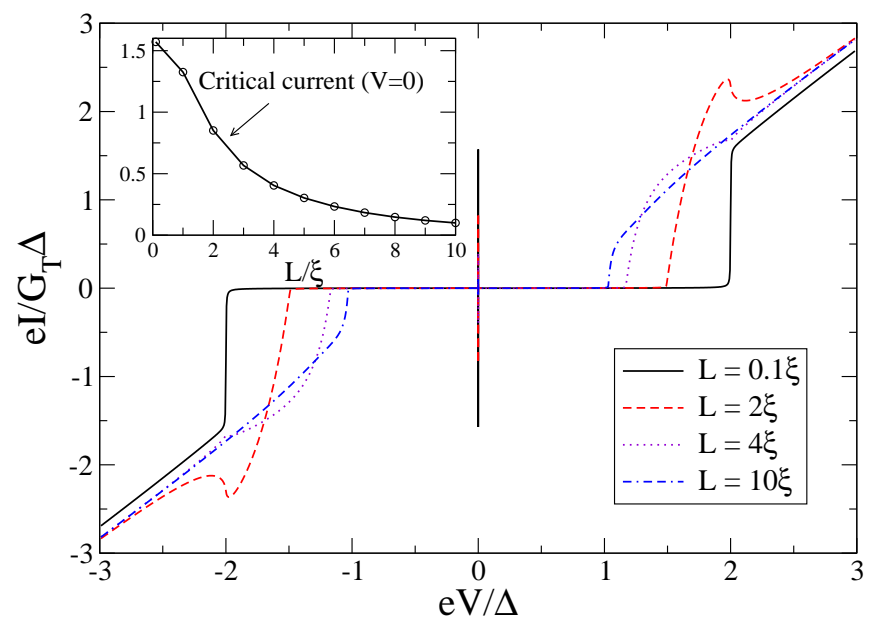

FIG. 2: (Color online) Zero-temperature dc current in the probe electrode $\mathrm{S}_{p}$ at zero bias $(U=0)$ for different lengths. The probe is located on $x=L / 2$. The inset shows the critical current as a function of the wire length.

\section{RESULTS AND DISCUSSION}

It is illustrative to start the discussion of the results with the case of zero-bias $(U=0)$. In this limit there are no Shapiro steps, but the dc current exhibits a supercurrent peak at $\mathrm{V}=0$. This can be seen in Fig. 2, where we plot the dc current through a probe located on $x=L / 2$ for different wire lengths. ${ }^{20}$ Apart from the supercurrent, the main feature of these I-V curves is the presence of a gap, which is equal to $\Delta+\Delta_{g}$, where $\Delta_{g}$ is the wellknown minigap present in the $\mathrm{N}$ wire due to proximity effect (see for instance Fig. 1 in Ref. 13). Such a minigap decays with $L$ as $\Delta_{g} \sim 3.2 \epsilon_{T}$, where $\epsilon_{T}=\hbar D / L^{2}$ is the Thouless energy. In the inset of Fig. 2 we show how the critical current decays with $L$. This critical current is a good reference for the height of the Shapiro steps at finite $U$. Notice in particular, that for $L \ll \xi$ the critical current adopts the value $I=\pi G_{T} \Delta / 2 e$, which is simply the critical current of a tunnel junction between BCS superconductors. 21

Now, we turn to the case of finite bias $U$. To give a first impression of how the steps would appear in an experiment, we show in Fig. 3)(a) the total contribution to the dc current, i.e. background current plus Shapiro steps, for $L=2 \xi, x=L / 2$ and three different values of the voltage $U$. Notice that since we are assuming that the system is voltage biased, the Shapiro steps appear as peaks in the current, rather than steps as in a current-biased contact. 2 We have also plotted in Fig. 3 both the distribution function and the non-equilibrium spectral function, which determine the shape of the background current. The first two peaks in the I-V at $V= \pm U / 2$ could simply be viewed as the supercurrent peaks that correspond to the condition of equality between the potentials of the probe $\mathrm{S}_{p}$ and of one of the reservoirs $\mathrm{S}_{l, r}$. However, their 

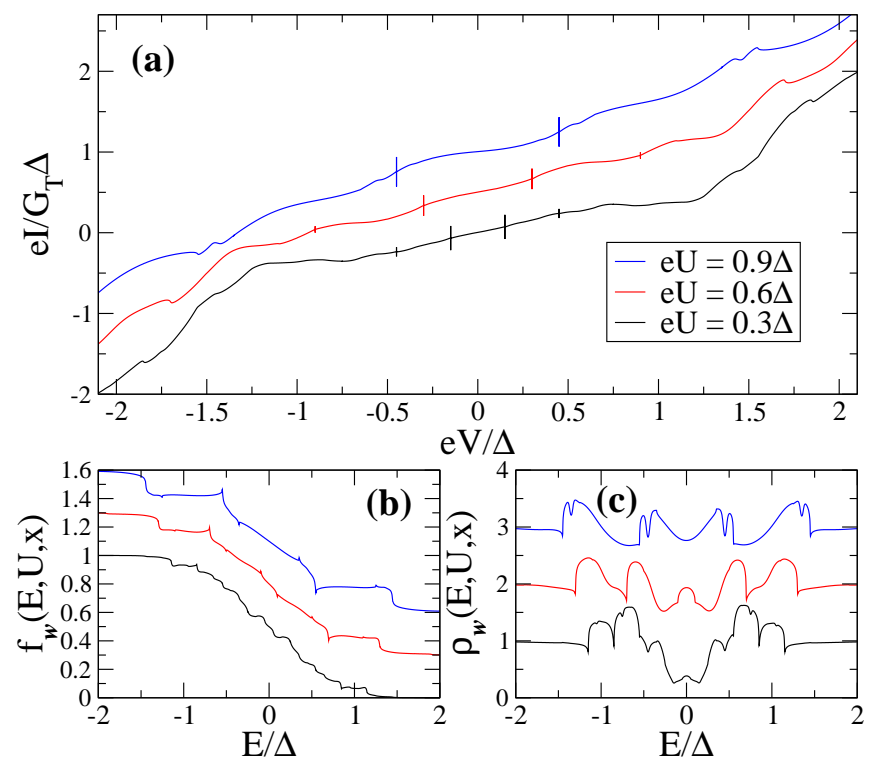

FIG. 3: (Color online) (a) Zero-temperature dc current in the probe $\mathrm{S}_{p}$ located on $x=L / 2$ for $L=2 \xi$ and three different values of the voltage $U$ : from bottom to top $e U / \Delta=$ $0.3,0.6,0.9$. (b) dc part of the distribution function of the normal wire at $x=L / 2$ as a function of the energy for the three voltages above. (c) Corresponding non-equilibrium spectral density in the normal wire $(x=L / 2)$ as a function of the energy. In the three panels the two upper curves have been shifted upwards for clarity.

height depends on $U$ and they are smaller than the critical current at $U=0$ because the quasiparticles in the wire are out of equilibrium. Notice, also, the large subgap current, which is absent in Fig. 2] More obvious manifestations of the interference of ac Josephson currents are the higher-order Shapiro steps, which appear at voltages that do not correspond to the alignment of the potential of $S_{p}$ to that of a reservoir. In Fig. 3(a), they are clearly seen at $V= \pm 3 U / 2$ at the lowest voltage $(U=0.3 \Delta)$, but they progressively disappear as $U$ increases.

Let us now study systematically the best conditions for the observation of the voltage-induced steps. First, we analyze their dependence on the wire length and the position of the probe at a given voltage $U$. In Fig. 4 we show for $e U=0.5 \Delta$ the height of the steps, denoted as $S_{m U / 2}$ for a step at $V=m U / 2$, as a function of $x$ for different wire lengths. Notice that we show the steps for positive voltages $V$ and the first one for negative voltages, $S_{-U / 2}$. Indeed, one can show that in this setup the following relation holds: $S_{-m U / 2}(x / L)=S_{m U / 2}(1-x / L)$ for $m>0$, which can be seen in the upper panels of Fig. 4. The most remarkable features of Fig. 4 are the following. First, the step $S_{U / 2}$ is of the same order as the critical current at $U=0$, although a bit smaller. Second, due to the biasing in this setup (see Fig. 1), the step $S_{U / 2}$ vanishes close the left electrode. The same hap-

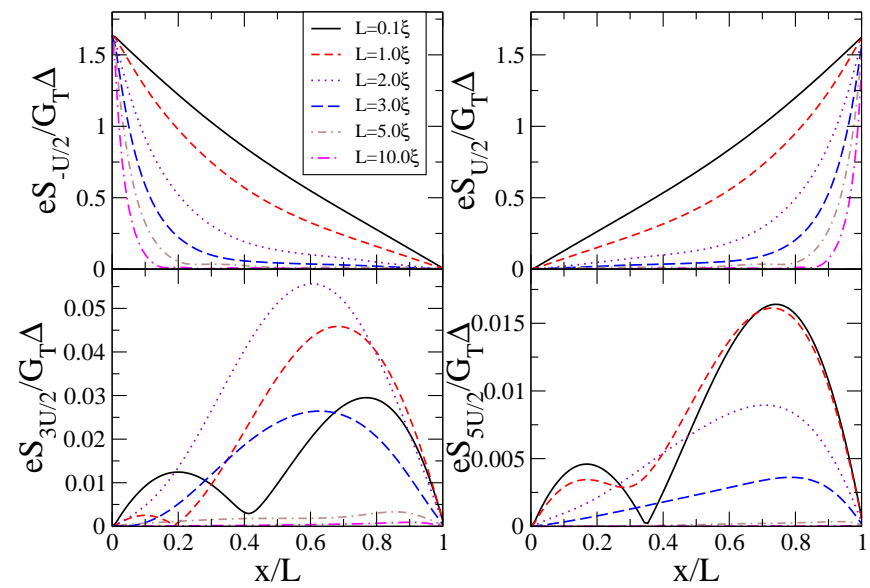

FIG. 4: (Color online) Height of the Shapiro steps at zero temperature as a function of the position of the probe $(x)$ for $e U=0.5 \Delta$ and different wire lengths.

pens with the steps $S_{3 U / 2}, S_{5 U / 2}, \ldots$ at both boundaries because we are assuming that the electrodes are ideal bulk reservoirs. Third, the higher-order steps $(m>1)$ are, for this particular voltage $U$, at least an order of magnitude smaller than $S_{U / 2}$. Finally, with respect to the length dependence, the steps progressively disappear as $L$ increases. For a given position, they decay roughly as $\xi / L$ for $L \gg \xi$, which is the usual decay of induced superconducting correlations in a normal diffusive system. However, for $L$ of the order of $\xi$, the dependence is not neccesarily monotonous, as it can be seen for the steps $S_{3 U / 2}$ and $S_{5 U / 2}$.

The other crucial parameter that controls the height of the steps is the voltage $U$. As Eq. (6) shows, in order to have a step at $V=m U / 2$ ( $m$ odd), one needs to have a non-zero component $\left[\mathcal{F}_{w}^{R, K}\right]_{0, m}$. Such an ac component is related to the coherent transfer of $n=(|m|+1) / 2$ Cooper pairs through the SNS junction, which requires the occurrence of coherent MARs of at least order $n$. An $n$-order process contributes significantly for voltages $2 \Delta / n<e U<2 \Delta /(n-1)$ and its probability decreases as the voltage increases. Thus, one naively expects (i) highorder $(m>1)$ steps to be more clearly visible at low bias $U$ and that (ii) at voltages $U>2 \Delta$, only the steps $S_{ \pm U / 2}$ survive. Indeed, these expectations are confirmed by the calculations, as can be seen in Fig. 5, where we show the height of the first two steps (for positive voltages) as a function of $U$ for different wire lengths and a probe located on $x=L / 2$. Notice that the step $S_{U / 2}$ reaches its maximum at $\sim 2 \Delta$ and then decays very slowly as the voltage increases whereas $S_{3 U / 2}$ vanishes rapidly for voltages above the gap. 


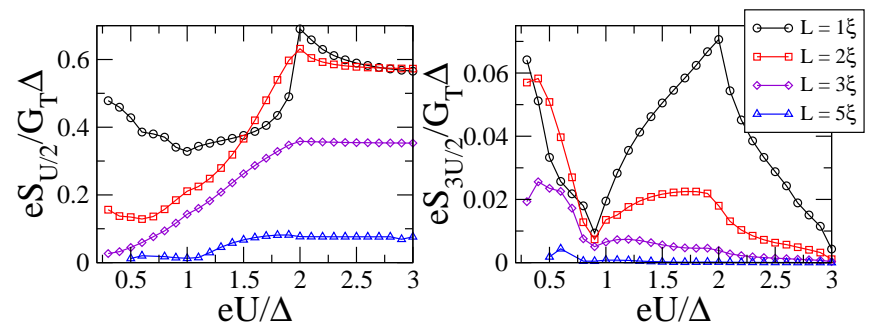

FIG. 5: (Color online) Height of the first two Shapiro steps for positive voltages in $x=L / 2$ at zero temperature as a function of $U$ for different lengths $L$.

\section{CONCLUSIONS}

In summary, we have predicted the possibility to generate parametrically Shapiro steps in multi-terminal superconducting structures by tuning the voltages in the reservoirs. We have illustrated this effect with detailed calculations for the case a diffusive SNS system with a third electrode coupled to the $\mathrm{N}$ region through a tunnel junction. Our results, based on the solution of the timedependent Usadel equations, show that these voltage- induced steps are a direct manifestation of the ac Josephson effect in the SNS junction and they are visible as long as there are sizable superconducting correlations induced in the $\mathrm{N}$ wire. We stress that these steps can be induced in any coherent mesoscopic structure attached to several (more than two) superconducting electrodes. Moreover, if in the setup of Fig. 1 all the electrodes were strongly coupled to the central wire, new phenomena would occur like the appearance of fractional Shapiro steps for $V=(m / n) U / 2$, with $m, n$ integers. Thus, the effect predicted here suggests the appearance of new physical phenonema and in particular, provides a new way to directly test the ac Josepshon effect in mesoscopic structures.

\section{Acknowledgments}

We acknowledge fruitful discussions with S. Bergeret, W. Belzig, D. Esteve, J.C. Hammer, A. Levy Yeyati, A. Martín-Rodero and C. Urbina. This work has been financed by the Spanish CYCIT (contract FIS2005-06255) and by the Helmholtz Gemeinschaft (contract VH-NG029).
1 B.D. Josephson, Phys. Lett. 1, 251 (1962).

2 S. Shapiro, Phys. Rev. Lett. 11, 80 (1963).

3 A. Barone and G. Paterno, Physics and Applications of the Josephson effect, John Wiley and Sons, Inc. (1982).

4 I. Giaever, Phys. Rev. Lett. 14, 904 (1965).

5 P.E. Lindelof and J. Bindslev Hansen, J. Low Temp. Phys. 29, 369 (1977).

${ }^{6}$ For a review see, J. Bindslev Hansen and P.E. Lindelof, Rev. Mod. Phys. 56, 431 (1984).

7 J.-X. Zhu, Z. Nussinov, and A.V. Balatsky, Phys. Rev. B 73, 064513 (2006).

${ }^{8}$ F. Pierre, A. Anthore, H. Pothier, C. Urbina, and D. Esteve, Phys. Rev. Lett. 86, 1078 (2001).

${ }^{9}$ For a review see, B. Pannetier and H. Courtois, J. Low Temp. Phys. 188, 599 (2000).

10 T.M. Klapwijk, G.E. Blonder and M. Tinkham, Physica B 109\&110, 1657 (1982).

11 J. Kutchinsky, R. Taboryski, T. Clausen, C.B. Sorensen, A. Kristensen, P.E. Lindelof, J. Bindslev Hansen, C. Schelde Jacobsen, and J.L. Skov, Phys. Rev. Lett. 78, 931 (1997); R. Taboryski, J. Kutchinsky, J. Bindslev Hansen, M. Wildt, C.B. Sorensen, and P.E. Lindelof, Superlattices Microstruct. 25, 829 (1999); T. Hoss, C. Strunk, T.
Nussbaumer, R. Huber, U. Staufer, and C. Schönenberger, Phys. Rev. B 62, 4079 (2000).

12 P. Dubos, H. Courtois, O. Buisson, and B. Pannetier, Phys. Rev. Lett. 87, 206801 (2001).

13 J.C. Cuevas, J.C. Hammer, J. Kopu, J.K. Viljas, and M. Eschrig, Phys. Rev. B 73, 184505 (2006).

14 A. Bardas and D. Averin, Phys. Rev. B 56, 8518 (1997).

15 E.V. Bezuglyi, E.N. Bratus, V.S. Shumeiko, G. Wendin, and H. Takayanagi, Phys. Rev. B 62, 14439 (2000).

16 K.D. Usadel, Phys. Rev. Lett. 25, 507 (1970).

17 M.Yu. Kuprianov and V.F. Lukichev, Sov. Phys. JETP 67, 1163 (1988).

18 One can show from Eq. (4) that only those ac components that involve the anomalous Green functions of the wire can give rise to Shapiro steps, i.e. components with an odd index $m$.

19 For this reason the steps were not observed in Ref. [8].

20 In Fig. 2 the phase difference between $S_{l}$ and $S_{r}$ is set to zero, which corresponds to the case where the supercurrent through the probe $\mathrm{S}_{p}$ reaches its maximum.

21 V. Ambegaokar and A. Baratoff, Phys. Rev. Lett. 10, 486 (1963). 